\title{
Optical sky brightness at Dome A, Antarctica, from the Nigel experiment
}

\author{
Geoff Sims $^{* a}$, Michael C. B. Ashley ${ }^{a}$, Xiangqun Cui ${ }^{b}$, Jon R. Everett ${ }^{a}$, LongLong Feng ${ }^{c, d}$, \\ Xuefei Gong ${ }^{b, d}$, Shane Hengst ${ }^{a}$, Zhongwen $\mathrm{Hu}^{b, d}$, Jon S. Lawrence ${ }^{e, f}$, Daniel M. Luong-van ${ }^{a}$, \\ Zhaohui Shang ${ }^{d, g}$, John W. V. Storey ${ }^{a}$, Lifan Wang ${ }^{c, h, d}$, Huigen Yang ${ }^{d, i}$, Ji Yang ${ }^{c}$, Xu Zhou ${ }^{d, j}$, \\ and Zhenxi Zhu ${ }^{c, d}$ \\ ${ }^{a}$ School of Physics, University of New South Wales, Sydney NSW 2052, Australia; \\ ${ }^{b}$ Nanjing Institute of Astronomical Optics \& Technology, Nanjing 210042, China; \\ ${ }^{c}$ Purple Mountain Observatory, Nanjing 210008, China; \\ ${ }^{d}$ Chinese Center for Antarctic Astronomy, China; \\ ${ }^{e}$ Department of Physics and Astronomy, Macquarie University, Sydney NSW 2109, Australia; \\ ${ }^{f}$ Australian Astronomical Observatory, Sydney NSW 1710, Australia; \\ ${ }^{g}$ Tianjin Normal University, Tianjin 300074, China; \\ ${ }^{h}$ Department of Physics and Astronomy, Texas A\&M University, College Station 77843, USA; \\ ${ }^{i}$ Polar Research Institute of China, Shanghai 200136, China; \\ ${ }^{j}$ National Astronomical Observatories, Chinese Academy of Science, Beijing 100012, China
}

\begin{abstract}
Nigel is a fiber-fed UV/visible grating spectrograph with a thermoelectrically-cooled $256 \times 1024$ pixel CCD camera, designed to measure the twilight and night sky brightness from $300 \mathrm{~nm}$ to $850 \mathrm{~nm}$. Nigel has three pairs of fibers, each with a field-of-view with an angular diameter of 25 degrees, pointing in three fixed positions towards the sky. The bare fibers are exposed to the sky with no additional optics. The instrument was deployed at Dome A, Antarctica in January 2009 as part of the PLATO (PLATeau Observatory) robotic observatory. During the 2009 winter, Nigel made approximately six months of continuous observations of the sky, with typically $10 \%$ deadtime between exposures. The resulting spectra provide quantitative information on the sky brightness, the auroral contribution, and the water vapour content of the atmosphere. We present details of the design, construction and calibration of the Nigel spectrometer, as well some sample spectra from a preliminary analysis.
\end{abstract}

Keywords: Dome A, PLATO observatory, site testing, aurora, night sky brightness

\section{INTRODUCTION}

Antarctica has long been recognised as an exceptional area for astronomical sites. ${ }^{1}$ Dome A, at a physical altitude of $4084 \mathrm{~m}$, is the highest point on the Antarctic plateau and is thought to rival other more established sites on the plateau (such as Dome C and the South Pole) due to its higher altitude, colder temperatures, lower wind speeds, and an atmospheric boundary layer that is confined very close to the ground..$^{2,3}$

Dome A was first visited via overland traverse by the Polar Research Institute of China (PRIC) in 2005, and, as of 2008 , hosts an array of site testing and scientific instruments on the PLATO robotic observatory. ${ }^{2}$ Nigel $^{4}$ is one such instrument, and was deployed in the summer of 2008-9 to measure and quantify the sky brightness - one of the most important considerations when evaluating the quality of available observing time at any given site.

The sky brightness is made up of a number of components, including scattered sunlight and moonlight, aurorae, airglow, zodiacal light, integrated starlight, as well as artificial sources. ${ }^{5}$ Spectrographic data collected from Nigel will be used to characterise the sky brightness above Dome A. The contributions and time varying intensity of the aurorae will be analysed, and the twilight sky will be profiled to determine when the sky becomes completely dark for various wavelengths, as well as to determine the precipitable water vapour content of the atmosphere.

\footnotetext{
* g.sims@student.unsw.edu.au
} 


\section{NIGEL}

Nigel uses six optical fibers (split into three pairs of "blue" and "red" optimised combinations) to obtain spectra from the night sky above Dome A in three fixed positions: North at $40^{\circ}$ altitude (chosen such that the Moon can pass through the fiber's field of view), West at $71.5^{\circ}$ altitude, and the zenith. The components and design of Nigel are described in detail in this section.

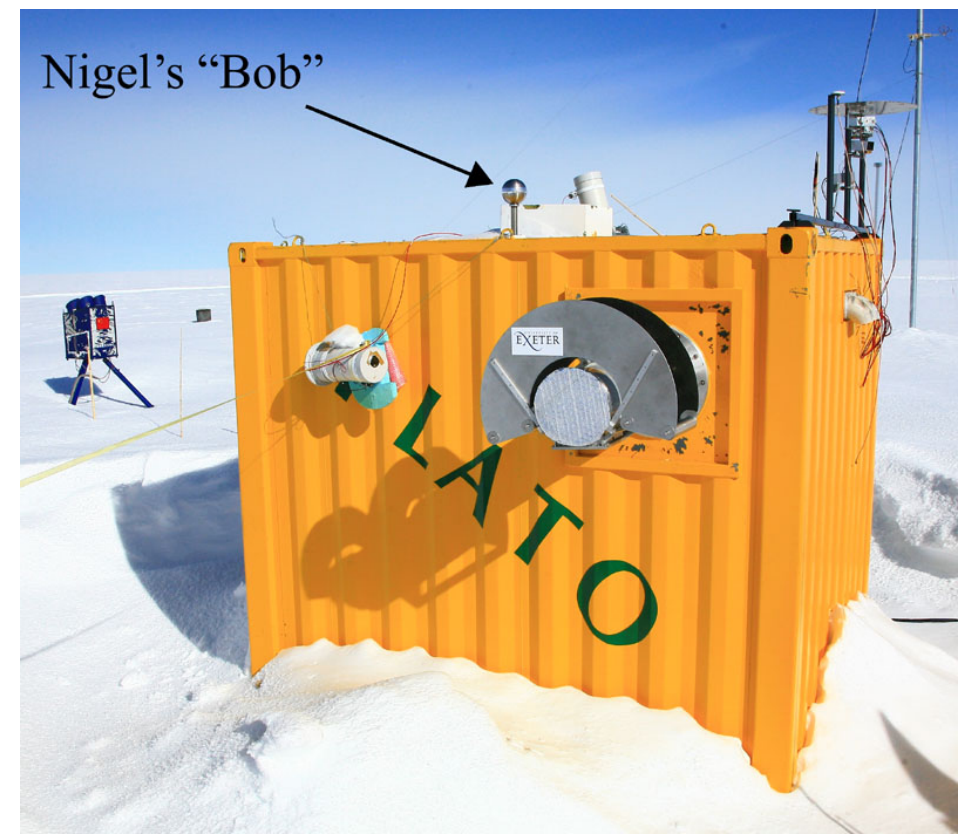

Figure 1: Nigel's "Bob", containing the input ends of the three pairs of optical fibers, on the roof of the PLATO observatory at Dome A, Antarctica, in January 2010. The snow accumulation around the base of PLATO is from one year of exposure.

\subsection{Bob Assembly}

It is crucial to the success of the Nigel instrument that the fibers are resistant to ice formation on their exposed faces. To achieve this, we mounted the fibers flush with the surface of a hollow stainless steel sphere. This has two important advantages: the sphere, being highly reflective, can be heated with a small amount of electrical power, and the spherical shape is resistant to build-up of snow.

The stainless steel sphere, also known as the "Bob" (see Figure 1), is $12 \mathrm{~cm}$ in diameter and constructed from two polished stainless steel hemispheres attached by an internal demountable collar which in turn is supported by a hollow stainless steel stalk.

The upper hemisphere contains three dual fiber end faces each consisting of an SMA connector stub drilled to fit two optical fibers and retained in a modified SMA bulkhead connector housing. The end of each fiber pair was inserted from the inside and oriented via pre-drilled holes at the three fixed positions on the sky. The fibers were then optically polished flush with the outer surface of the upper hemisphere.

The three assemblies are also attached to internal copper plates which can be electrically heated via current flowing through two $25 \Omega 10 \mathrm{~W}$ resistors. This arrangement ensures the fiber end faces are kept clear of frost or ice.

The Bob is supported by a hollow stalk through which the six fiber patch cords pass into the instrument module. Each patch cord is terminated on a bulkhead which also supports six collimating filter assemblies (where the light from the "red" fibers passes through a high-pass filter, as described in Section 2.2). The fibers are then 
aligned and bonded to a precision $\mathrm{V}$ block, with an optically polished output face, and attached to a connector to create a pseudo-slit at the input of the spectrometer.

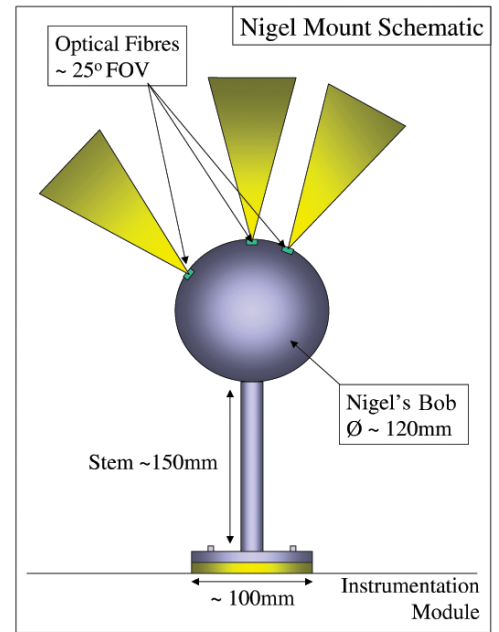

Figure 2: Detail of Nigel's "Bob". The shaded triangles represent the fields-of-view of the three pairs of fibers.

\subsection{Fibers and Filters}

Step-index multimode fibers from Ocean Optics Ltd were selected for use in Nigel. The fibers have a diameter of $100 \mu \mathrm{m}$ and a quoted numerical aperture (NA) of 0.22 corresponding to a field-of-view with an angular diameter of $25^{\circ}$.

The concentration of hydroxyl $(\mathrm{OH})$ within a fiber determines the fiber's spectral attenuation profile. ${ }^{6}$ Fibers with high concentrations of $\mathrm{OH}$ are most efficient from 300-1100 nm (although with a deep absorption at $\sim 950 \mathrm{~nm}$ ), while those with lower $\mathrm{OH}$ are typically used at longer wavelengths. Of the six fibers, three were chosen to be high-OH (corresponding to the blue end of the spectrum), and three were chosen to be low-OH (for the red end of the spectrum).

To avoid contamination from the second order of the grating, the low-OH ("red") fibers were used in conjunction with a Schott OG515 filter, which has long-pass filtering at $515 \mathrm{~nm}$.

\subsection{Spectrograph and CCD Camera}

The spectrograph used is a Jobin Yvon model CP200 (1989) holographic diffraction grating, with 200 grooves/mm. The focal length is $190 \mathrm{~mm}$ and magnification is 1:1. The spectra are recorded using an Oriel Instruments model Instaspec IV thermo-electrically cooled CCD camera, which has an exposure area of $256 \times 1024$ pixels. Our achieved resolution is $3 \mathrm{~nm}$ FWHM at $500 \mathrm{~nm}$. Shutter-less operation was used, with the spectra being rapidly shifted into the unused lower half of the CCD at the end of each exposure.

\subsection{Software and control}

The Nigel CCD was interfaced to an ISA card connected to a PC/104 computer running MS-DOS 6.22 and ERIC 7 software. The instrument was in turn controlled by the PLATO Supervisor computer running Debian Linux. Exposure times were set based on the results from the immediately preceeding exposure, to avoid saturation on the sky. Due to the limited communications bandwidth, the CCD images were analysed at Dome A. The images were dark-subtracted and multiplied by a mask to optimally extract the spectra (i.e., the spectra were weighted on the basis of their signal-to-noise). The six 2D spectra on each image were then collapsed to 1D and this information was sent out via Iridium satellite modem. The original raw images were stored on solid-state disks, and have now been retrieved by the $2009 / 2010$ Chinese traverse team. 


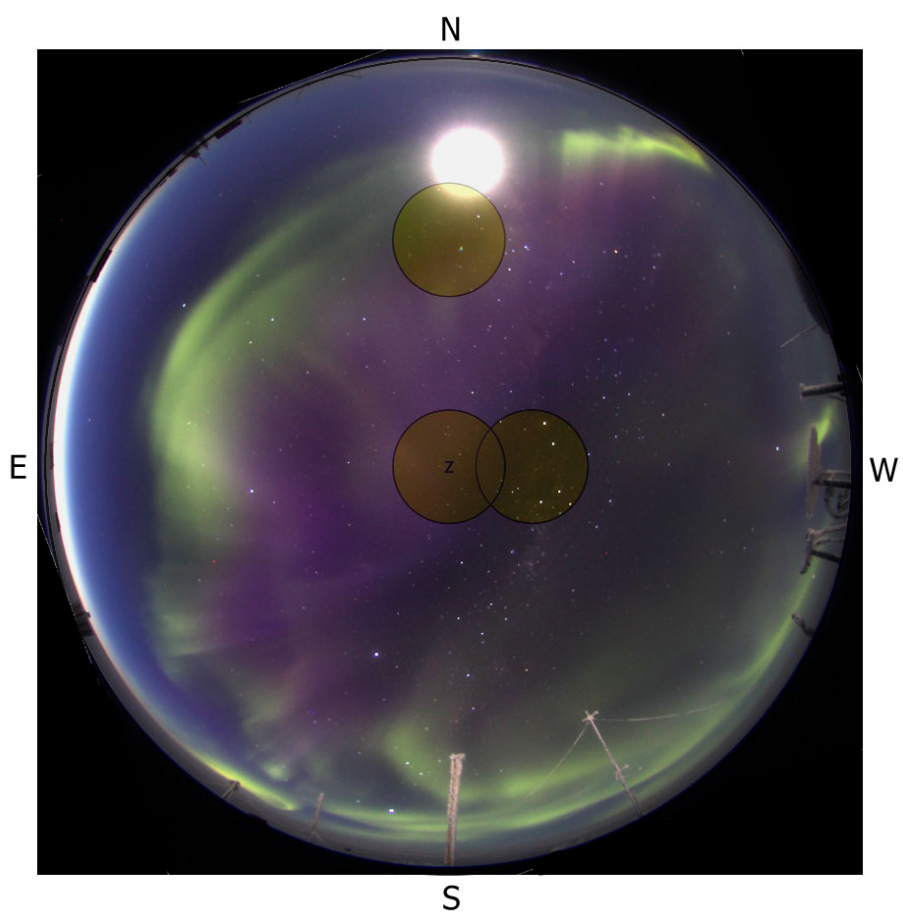

Figure 3: Nigel's approximate fields of view, overlaid with a photograph from HRCAM (an all-sky camera, also on the PLATO suite at Dome A), during a strong auroral display on April 5, 2010. The white disk is the moon. The cardinal directions are approximately shown.

\section{CALIBRATION}

\subsection{Wavelength Calibration}

The spectra were wavelength-calibrated using ten known Fraunhofer absorption lines in the blue sky spectra, and five auroral and airglow lines in the dark sky spectra. The actual spectra used for the calibration were a combination of many observations, averaged in order to increase the signal-to-noise ratio and make the spectral features easier to centroid.

\subsection{Flux Correction and Calibration}

The various components of Nigel (optical fibers, Schott filter, spectrograph and the CCD camera) each have their own spectral response, as shown in Figure 4. Figure 5 shows the convolution of all the components, and represents our anticipated overall spectral response.

We aim to perform an absolute flux calibration by comparing the uncalibrated flux data from Nigel with calibrated flux data from the Gattini ${ }^{8}$ camera (also part of the PLATO suite of instruments at Dome A). The Gattini camera points to the zenith, and covers a very wide angle (90 degrees) which encompasses the entire field-of-view of the Nigel zenith fibers. Gattini uses well defined astronomical filters, and can be absolutely calibrated by performing photometry on reference stars. By carefully selecting a region corresponding to the field of view of the Nigel zenith fiber, an accurate absolute flux calibration can be achieved.

\section{PRELIMINARY RESULTS}

\subsection{Example Spectra}

Representative spectra of the twilight sky and strong auroral events are reproduced in Figures 6 and 7 , respectively. A total of 1000 observations were averaged for the twilight spectrum, and 86 observations for the auroral 


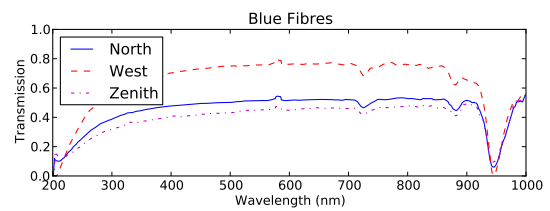

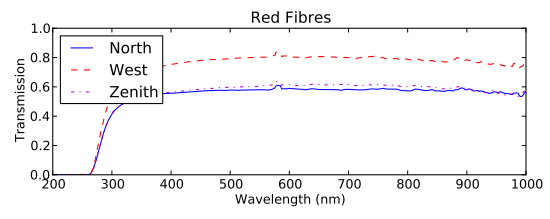

(a) Fibers

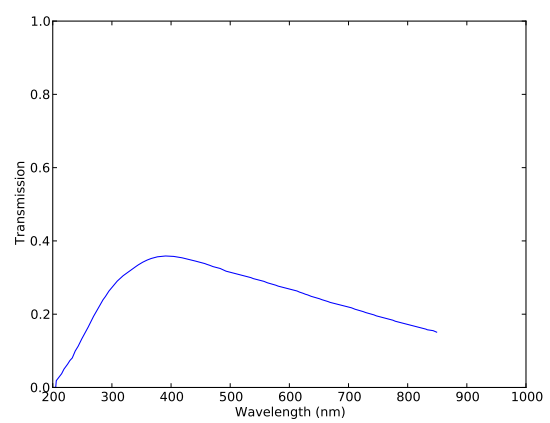

(c) Spectrograph

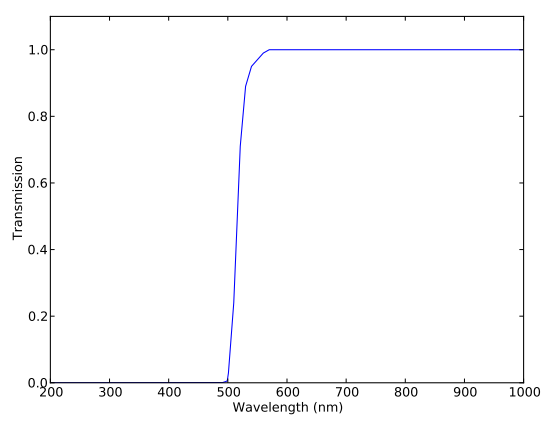

(b) Schott $515 \mathrm{~nm}$ Filter

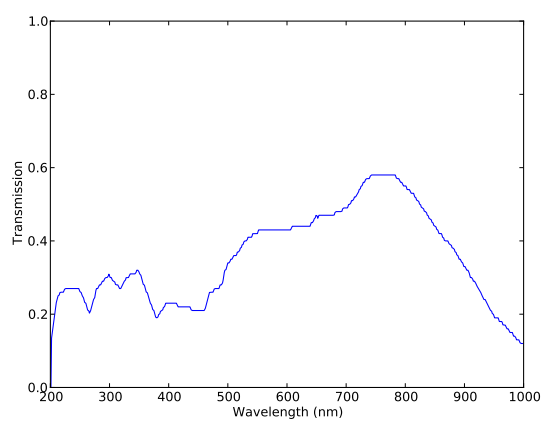

(d) CCD camera

Figure 4: Instrumental spectral response curves. The spectrograph response is only known between 300 and $850 \mathrm{~nm}$.

spectrum, to increase the signal-to-noise ratio and to make features more readily identifiable.

\subsection{Astronomical Darkness}

Another important consideration when selecting an observing site is the amount of available "dark time". At polar latitudes, this is strongly dependent on the limit set by the solar zenith distance at which the sky becomes completely dark. Formally, the time at which an astronomical site is considered to be "dark" is defined as when the Sun is greater than $18^{\circ}$ below the horizon (or equivalently, the solar zenith distance $\left(Z D_{\odot}\right)$ is greater than $\left.108^{\circ}\right)$. The darkness of the twilight sky depends on the aerosol content of the atmosphere, ${ }^{9}$ and, as Dome A has an exceptionally clear atmosphere with no dust or haze, we might expect to use a smaller limit for $Z D_{\odot}$, and hence recover a substantial amount of dark time.

Spectra from Nigel allow us to estimate the practical limit for $Z D_{\odot}$ as a function of wavelength. For example, by convolving Nigel spectra with a Bessell $\mathrm{V}$ band filter profile we can generate the data shown in Figure 8 . Table 1 lists various solar zenith distances and the corresponding amount of dark time available at Dome A.

\section{FUTURE WORK}

Spectra from Nigel will allow us to identify and confirm auroral events, providing us with detailed statistics about the intensity and spatial distribution of auroral activity from Dome A. Additionally, we will determine the relationship between sky brightness and solar zenith distance, as a function of wavelength, and compare it with other observatories such as ESO-Paranal. ${ }^{10}$ We should also be able to derive the water vapour content of the atmosphere. 

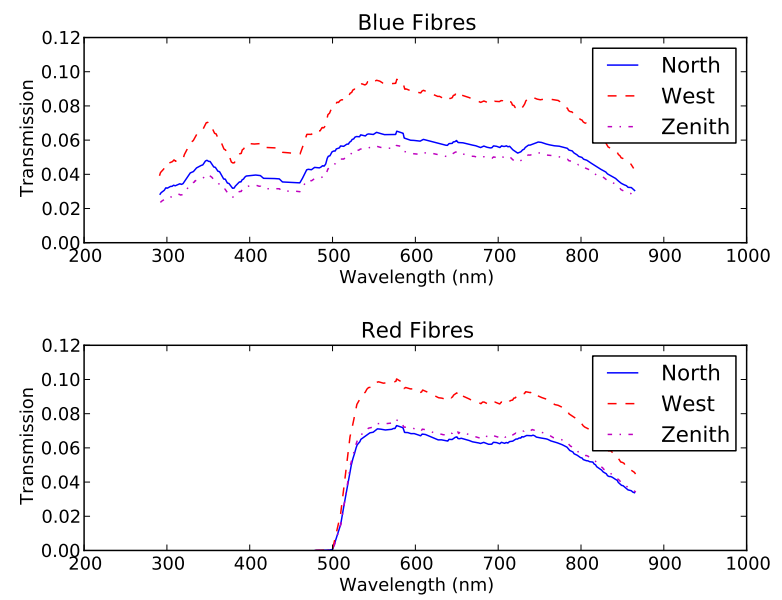

Figure 5: Predicted overall response for each of the six channels.

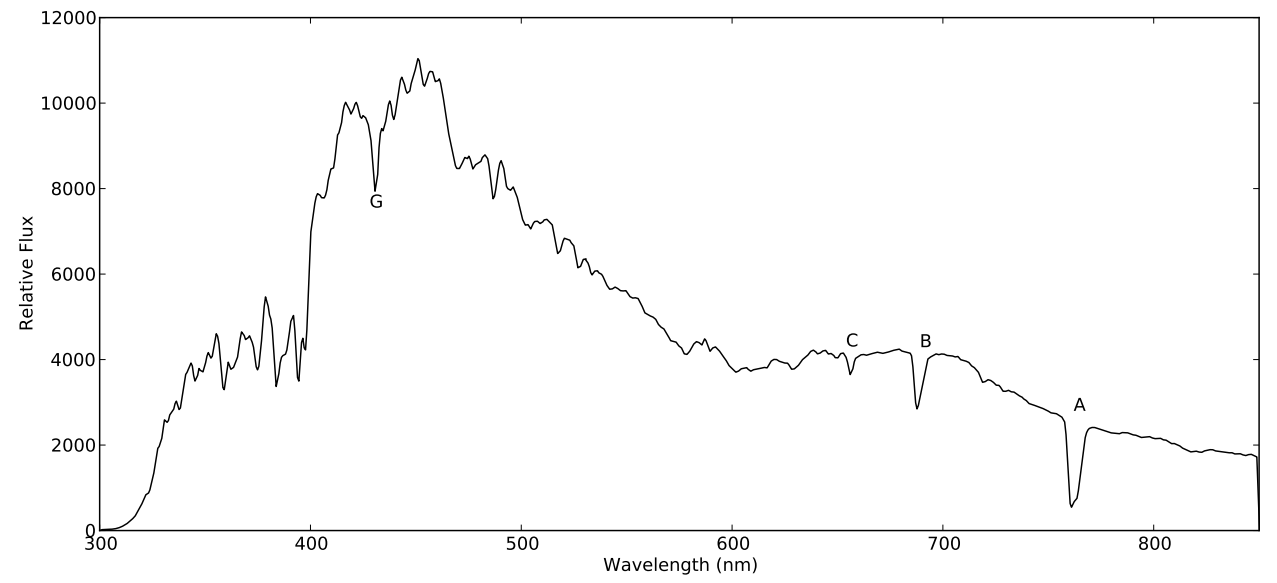

Figure 6: Typical spectrum of the twilight sky from Dome A, with approximate flux calibration using the predicted instrumental responses from Figure 5. We note that the flux calibration is particularly uncertain in the blue. This spectrum is a composite of red and blue fiber spectra, spliced at $\sim 530 \mathrm{~nm}$. A number of Fraunhofer absorption bands are visible: A $(759.4 \mathrm{~nm})$ and B $(686.7 \mathrm{~nm})$ due to molecular oxygen, $\mathrm{C}(656.3 \mathrm{~nm})$ due to hydrogen- $\alpha$, and $\mathrm{G}(430.8 \mathrm{~nm})$ from iron. It is noteworthy that the water vapour absorption features at 730 and $820 \mathrm{~nm}$ - normally quite deep at temperatelatitude observatories - are almost entirely absent at Dome A, due to the exceedingly low water vapour content of the atmosphere.

\section{ACKNOWLEDGMENTS}

This research is supported by the Chinese PANDA International Polar Year project and the Polar Research Institute of China. The authors wish to thank all the members of the 2008/2009/2010 PRIC Dome A expeditions for their heroic efforts in reaching the site and for providing invaluable assistance to the expedition astronomers in setting up the PLATO observatory and its associated instrument suite. This research is financially supported by the Australian Research Council, the Australian Antarctic Division, the Chinese Academy of Sciences, the European Commission Sixth Framework Program, the National Natural Science Foundation of China, the US 


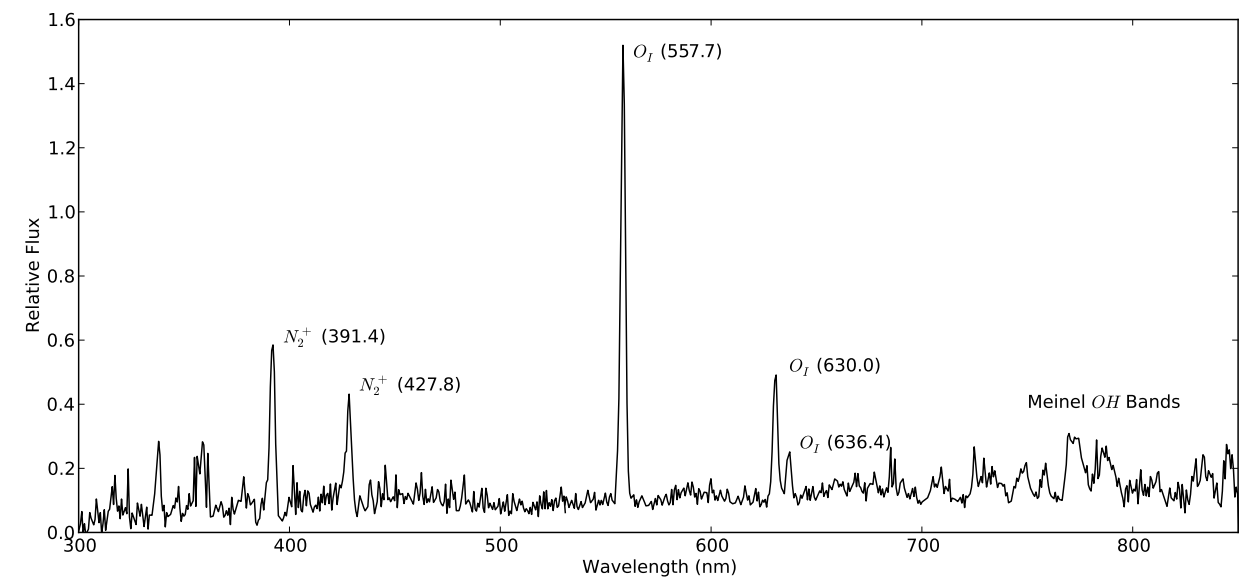

Figure 7: Average of 86 spectra with strong auroral lines, as observed from Dome A. The individual red and blue fiber spectra were spliced at $\sim 530 \mathrm{~nm}$, and roughly flux-calibrated using the predicted instrumental responses from Figure 5. We see two prominent emission lines corresponding to molecular nitrogen $\mathrm{N}_{2}$ at wavelengths $391.4 \mathrm{~nm}$ and $427.8 \mathrm{~nm}$. The three other significant emissions lines are attributed to excited oxygen atoms at $557.7 \mathrm{~nm}$ (also dominant in airglow emission spectra), $630.0 \mathrm{~nm}$ and $636.4 \mathrm{~nm}$. The features visible for wavelengths longer than $650 \mathrm{~nm}$ are the Meinel rotation-vibration bands of $\mathrm{OH}$.

Table 1: Amount of available dark time for various solar zenith distances.

\begin{tabular}{cc}
\hline$Z D_{\odot}$ & Dark Time (hours) \\
\hline \hline 108 & 1678 \\
107 & 1827 \\
106 & 1987 \\
105 & 2161 \\
104 & 2367 \\
103 & 2603 \\
102 & 2787 \\
\hline
\end{tabular}

National Science Foundation, and the United States Antarctic Program. Additional financial contributions have been made by the institutions involved in this collaboration.

We thank Anna Moore for critical comments on the draft of this manuscript.

\section{REFERENCES}

[1] Lawrence, J. S., Ashley, M. C. B., Tokovinin, A. and Travouillon, T., "Exceptional astronomical seeing conditions above Dome C in Antarctica," Nature, 431, 278-281 (2004).

[2] Yang, H., Allen, G., Ashley, M. C. B., Bonner, C. S., Bradley, S., Cui, X., Everett, J. R., Feng, L., Gong, X., Hengst, S., Hu, J., Jiang, Z., Kulesa, C. A., Lawrence, J. S., Li, Y., Luong-Van, D., McCaughrean, M. J., Moore, A. M., Pennypacker, C., Qin, W., Riddle, R., Shang, Z., Storey, J. W. V., Sun, B., Suntzeff, N., Tothill, N. F. H., Travouillon, T., Walker, C. K., Wang, L., Yan, J., Yang, J., York, D., Yuan, X., Zhang, X., Zhang, Z., Zhou, X. and Zhu, Z., "The PLATO Dome A Site-Testing Observatory: Instrumentation and First Results", Publ. Astron. Soc. Pac., 121, 174-184 (2009). 


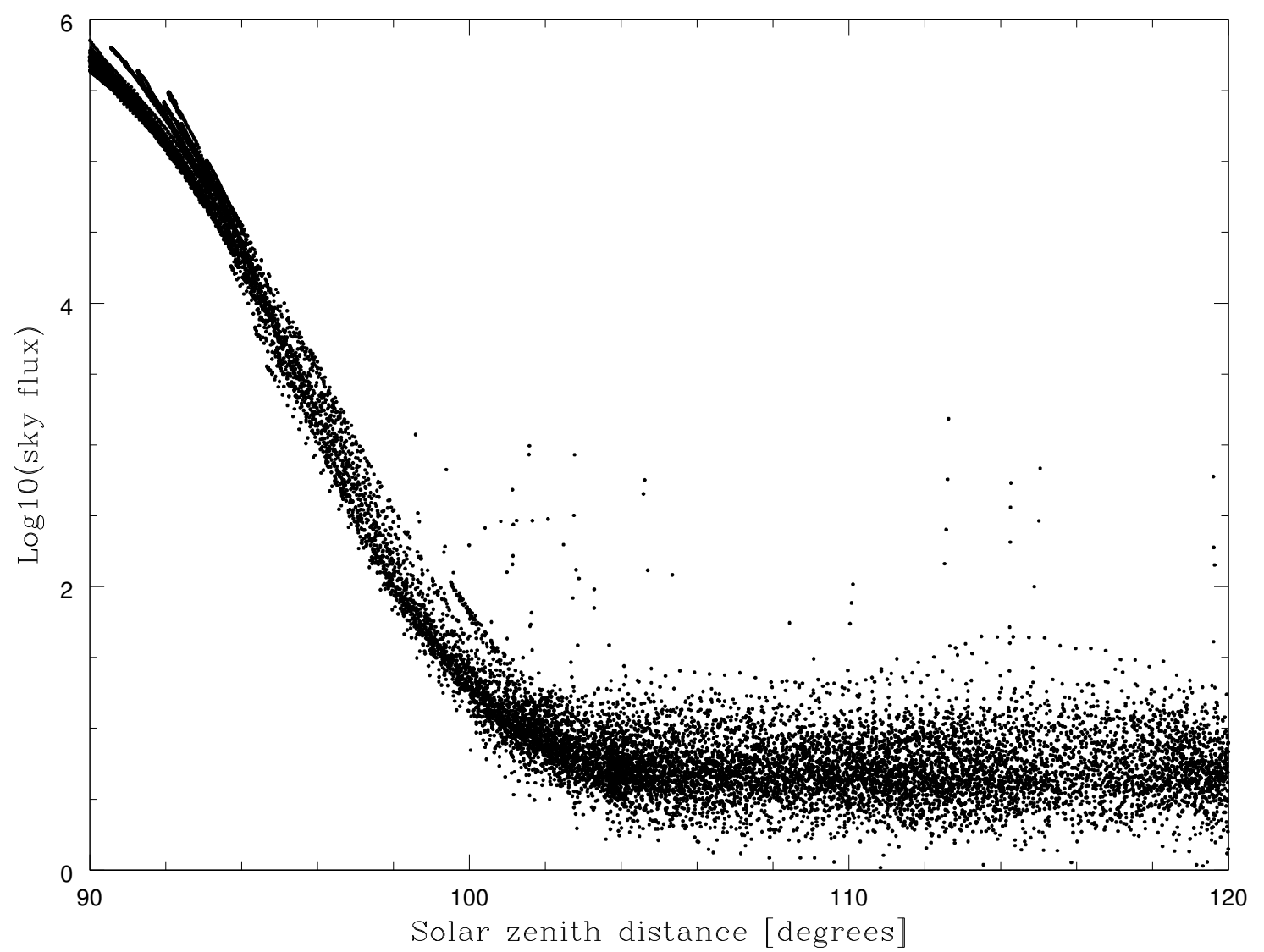

Figure 8: Zenith optical sky brightness in the Bessell $\mathrm{V}$ band as a function of solar zenith distance. Each point is the result of convolving a single Nigel spectrum with a V-band filter profile. Fluxes are in arbitrary units. Data excludes times when the moon was up. The points that are anomalously high are probably due to aurorae. The spread of points at low fluxes is due at least in part to hitting the noise floor of the instrument. The limit for astronomical twilight is formally $Z D_{\odot}=108^{\circ}$; a practical limit derived from this data is $Z D \odot \sim 103^{\circ}$.

[3] Bonner, C. S., Ashley, M. C. B., Cui, X., Feng, L., Gong, X., Lawrence, J. S., Luong-Van, D. M., Storey, J. W. V., Wang, L., Yang, H., Yang, J., Zhou, X. and Zhu, Z., "Height of The Atmospheric Boundary Layer Above Dome A, Antarctica, during 2009," Publ. Astron. Soc. Pac., submitted (2010).

[4] Kenyon, S. L., Ashley, M. C. B., Everett, J., Lawrence, J. S. and Storey, J. W. V., "Nigel and the optical sky brightness at Dome C, Antarctica," Proc. SPIE, 6267, 62671M (2006).

[5] Kenyon, S. L. and Storey, J. W. V., "A review of optical sky brightness and extinction at Dome C, Antarctica", Publ. Astron. Soc. Pac., 118, 489-502 (2006).

[6] Ocean Optics, "Optical Fiber Attenuation Spectra", http://www.oceanoptics.com/Products/fiberattenuation.asp (2010).

[7] Ashley, M. C. B., Brooks, P. W., and Lloyd, J. P., "Remote control of astronomical instruments via the Internet", Publ. Astron. Soc. Australia, 13, 17-21 (1996).

[8] Moore, A., Allen, G., Aristidi, E., Ashley, M., Bedding, T., Beichman, C., Briguglio, R., Busso, M., Candidi, M., Ciardi, D., Cui, X., Cutispoto, G., Distefano, E., Espy, P., Everett, J., Feng, L., Hu, J., Jiang, Z., 
Kenyon, S., Kulesa, C., Lawrence, J., Le Roux, B., Leslie, T., Li, Y., Luong-Van, D., Phillips, A., Qin, W., Ragazzoni, R., Riddle, R., Sabbatini, L., Salinari, P., Saunders, W., Shang, Z., Stello, D., Storey, J., Sun, B., Suntzeff, N., Taylor, M., Tosti, G., Tothill, N., Travouillon, T., Van Belle, G., Von Braun, K., Wang, L., Yan, J., Yang, H., Yuan, X., Zhu, Z. and Zhou, X., "Gattini: a multisite campaign for the measurement of sky brightness in Antarctica", Proc. SPIE, 7012, 701226-701226-10 (2008).

[9] Ugolnikov, O., Postylyakob, O. V. and Maslov, I. A., "Effects of multiple scattering and atmospheric aerosol on the polarization of the twilight sky," J. Quant. Spectrosc. Radiat. Transfer, 88, 233-241 (2004).

[10] Patat, F., Ugolnikov, O. and Postylyakov, O. V., "UBVRI twilight sky brightness at ESO-Paranal," Astron. Astrophys., 455, 385-393 (2006). 